\title{
El control del cannabis: de las políticas nacionales al régimen global
}

\author{
ANDRÉs LÓPEZ RESTREPO*
}

Artículo recibido: 27 de marzo de 2020

Artículo aprobado: 21 de mayo de 2020

Doi: https://doi.org/10.12804/revistas.urosario.edu.co/desafios/a.8855

Para citar este artículo: López, A. (2021). El control del cannabis: de las políticas nacionales al régimen global. Desafíos, 33(1), 1-29. https://doi.org/10.12804/revistas.urosario.edu.co/ desafios/a.8855

\section{Resumen}

Cuando el régimen internacional de control de drogas apareció a principios del siglo XX tenía como objetivo ocuparse del opio y sus derivados. En los años siguientes incluyó algunas drogas, como la cocaina, y excluyó otras, como el alcohol. El cannabis babía sido probibido antes de la creación del régimen internacional en diversos países del mundo musulmán, el Africa subsahariana y América Latina, ninguno de los cuales era decisivo en la comunidad internacional. El cannabis fue incluido en el régimen en 1925 poriniciativa de Egipto, Sudáfrica y la India, y su probibición definitiva apenas tuvo lugar en 1961.

Palabras clave: politica sobre drogas; consumo de drogas; cannabis; maribuana; bistoria.

\footnotetext{
* Universidad Nacional de Colombia (Bogotá). Correo electrónico: alopezre@unal.edu.co. ORCID: http://orcid.org/0000-0002-2649-2571
} 


\title{
Cannabis Control: From National Policies to the Global Regime
}

\begin{abstract}
The international drug control regime appeared in the early $20^{\text {th }}$ century with the purpose of dealing with opium and its derivatives. In the following years, it included some drugs, such as cocaine, and excluded others, such as alcohol. Cannabis had been banned before the creation of the international regime in various countries of the Muslim world, sub-Saharan Africa and Latin America, none of which was decisive in the international community. Cannabis was included in the regime in 1925 at the initiative of Egypt, South Africa and India and its definitive probibition only took place in 1961.
\end{abstract}

Keywords: Drug policy; drug consumption; cannabis; marijuana; history.

\section{O controle da cannabis: das políticas nacionais ao regime global}

\begin{abstract}
Resumo
Quando o regime internacional de controle de drogas apareceu no começo do século XX tinha como objetivo se ocupar do ópio e seus derivados. Nos anos a seguirincluiu algumas drogas, como a cocaína, e excluiu outras, como o álcool. A cannabis tinha sido proibida antes da criação do regime internacional em diversos países do mundo muculmano, a África subsaariana e a América Latina, nenbum dos quais era decisivo na comunidade internacional. A cannabis foi incluída no regime em 1925 por iniciativa do Egito, a África do Sul e a Índia, e sua proibição definitiva apenas teve lugar em 1961.
\end{abstract}

Palavras-chave: politica sobre drogas; consumo de drogas; cannabis; marijuana; bistória.

\section{Introducción}

Somos testigos en estos tiempos de un cambio acelerado en el estatus legal del cannabis, la droga ilícita más consumida en todo el mundo. Su descriminalización, incluso legalización, se han hecho realidad en 
diversas partes del planeta y otras más transitan por el mismo sendero. Es razonable que así sea, teniendo en cuenta que el cannabis es menos nocivo que otras drogas, tanto legales - alcohol y tabacocomo ilegales —opiáceos y cocaína- De ninguna manera se trata de una droga inocua; por el contrario, su uso está relacionado con problemas mentales y puede afectar el aprendizaje y el coeficiente intelectual de los adolescentes. Sin embargo, la proporción de sus consumidores que pueden considerarse dependientes es inferior a la del alcohol y el tabaco, y, a diferencia de estas dos drogas, su uso no tiene consecuencias fatales.

Surge entonces la pregunta de por qué fue prohibido el cannabis. El régimen internacional de control de drogas apareció a principios del siglo Xx con el propósito de resolver el problema del opio para fumar en China. Así, la Comisión de Shanghái de 1909, la reunión precursora de ese régimen, se ocupó del opio y sus derivados. La Convención del Opio de 1912, que adoptó las primeras resoluciones vinculantes sobre el tema de las drogas, incluyó además la cocaína, revelando así el carácter expansivo del régimen. El cannabis entró a hacer parte del régimen con la Convención del Opio de 1925 y la amapola y la hoja de coca fueron incorporadas finalmente mediante la Convención Única de Estupefacientes de 1961, que además prohibió de manera definitiva el cannabis. No obstante, identificar la tendencia del régimen a incluir una creciente cantidad de drogas no da ninguna pista sobre las sustancias que incluye o excluye. Esto ha dependido de muchos factores. Era inevitable que el opio fuese seguido por sus derivados como la morfina y la heroína y demás opiáceos, pues eran más potentes que el mismo opio y, por lo tanto, la regulación parecía aún más necesaria. Dos de las drogas más nocivas, el alcohol y el tabaco, quedaron al margen por razones que Courtwrigth (2001) examina en detalle. La cocaína causó mucha preocupación a fines del siglo XIX y se consideró que podía ser tan adictiva como los opiáceos, por lo que fue inevitable que fuese sometida al mismo régimen que el opio y sus derivados. En cambio, lo ocurrido con las plantas que eran consumidas de manera directa o servían de materia prima para fabricar otras drogas fue un proceso más complejo. El cannabis fue incluido en el régimen por iniciativa de unos pocos países y hubo que esperar varias décadas para que la hoja 


\section{I ANDRÉs López Restrepo}

de coca y la amapola hiciesen parte. Este texto analiza el proceso por el cual el cannabis llegó a ser incluido en el régimen.

Hasta no hace mucho tiempo estaba muy extendida la idea de que la influencia y presión de Estados Unidos fueron los factores determinantes en esa prohibición, como afirman Abel (1980, pp. 242 y 254-255) y Booth (2003, pp. 249-250). Antonio Escohotado, autor de Historia general de las drogas, la síntesis más popular sobre el tema en lengua española, dice en otro texto: "Estados Unidos fue el primer país que prohibió —en vez de simplemente controlar (para 'usos médicos y científicos') — el hachís y la marihuana, mediante una ley de 1937 que sólo afectó al principio a pequeñas minorías de negros y latinoamericanos" (1997, p. 28). Monografías más recientes, que hacen un uso atento de los archivos, descartan esa interpretación y muestran que muchos países prohibieron el cannabis antes que Estados Unidos y sin su influencia. Ejemplos de ese tipo de trabajo son Campos (2012), Kozma (2011) y Mills (2003, 2013). Sin embargo, no existe un trabajo comparado que retome los resultados de esos estudios de caso para presentar un panorama general que dé cuenta del paso de las prohibiciones nacionales a un régimen global.

Este artículo aprovecha los resultados de esas monografías recientes y presenta por primera vez el proceso por el cual la droga llegó a ser regulada y prohibida, primero en el ámbito nacional, en los países líderes en el control del cannabis, y luego en el internacional. La primera parte presenta la historia de los diversos usos de la planta en las distintas regiones del mundo. En la segunda sección se examina cómo fue regulado y prohibido el cannabis en algunos países, y cuáles fueron las razones que se arguyeron para hacerlo. El tercer apartado expone cómo se incluyó el cannabis dentro del régimen internacional de control de drogas. En la conclusión se discuten la reciente flexibilización del estatus legal del cannabis y sus perspectivas de futuro. El texto presenta lo ocurrido con el cannabis desde una perspectiva global, pero presta particular atención a lo sucedido en América Latina y en Colombia. 


\section{La globalización de la planta}

El cannabis es una de las plantas más resistentes y ubicuas que existen. Se da de manera silvestre en muchos lugares y ha sido cultivada desde hace miles de años. El cannabis es originario del centro de Asia, donde la formación del Himalaya separó dos poblaciones de plantas que evolucionaron de manera paralela hasta dar lugar a dos especies distintas, el Cannabis sativa y el Cannabis indica, cuyas diferencias fueron ampliadas después por la selección humana. La especie sativa, característica de Europa, es más alta, con ramas muy separadas y es usada para la producción de fibra y semillas. Se denomina cáñamo al cannabis cultivado para fibra, que es resistente y duradera, y las semillas, llamadas cañamón, sirven para alimentar aves y para fabricar un aceite rico en grasas y proteínas. $\mathrm{El}$ C. indica, como su nombre lo indica, es del subcontinente indio y es más pequeño y frondoso, y es la especie usada por sus efectos psicoactivos. El tetrahidrocannabinol (THC), que es el elemento psicoactivo más importante del cannabis, es un alucinógeno suave que sirve además como analgésico, relajante muscular, antidepresivo y antiemético, y reduce ataques epilépticos, dilata el tejido bronquial y afecta el apetito. Las dos especies de cannabis contienen THC, pero solo se encuentra en cantidades significativas en el C. indica (Schultes, Klein, Plowman \& Lockwood, 1974, pp. 337-367; Duvall, 2015, pp. 9-32 y 89-91; Duvall, 2017a). Quienes consumen cannabis por sus efectos psicoactivos lo hacen de tres formas diferentes: como hachís, que es hecho de la resina o las glándulas resineras de la planta hembra; como marihuana, que comprende las hojas y flores secas del cannabis; y, raramente, como aceite de hachís, que es un concentrado líquido extraído mediante procedimientos químicos de la resina. La marihuana contiene del $5 \%$ al $15 \%$ de THC, el hachís hasta un 20\% y puede llegar a un $85 \%$ en el caso del aceite (Booth, 2003, pp. 7-18).

Los cultivos más antiguos de C. indica han sido encontrados en China y se remontan a los primeros momentos de su civilización. Los chinos conocieron sus efectos psicoactivos, pero no tuvieron mucho interés en ellos y prefirieron usar la planta como alimento y medicina, y, sobre todo, como fibra y para hacer papel, el cual inventaron. 


\section{6 / ANDRÉs López Restrepo}

El consumo psicoactivo del C. indica apareció hacia el año 2000 a. C. en la zona fronteriza entre Afganistán, Pakistán y Tayikistán. De allí se extendió hacia la India, que es el país con la tradición más antigua y compleja del cannabis, y fue su mayor productor y consumidor en el mundo hasta la década de 1960, cuando su uso se popularizó en Estados Unidos, que se convirtió desde entonces en el principal mercado del producto. La planta se ha empleado en la India como medicina, para ayudar a sobrellevar las fatigas del trabajo físico, como acompañamiento de todo tipo de rituales religiosos y culturales, y por simple placer, por gentes de todas las condiciones, tanto musulmanes e hindúes, del campo y la ciudad, pero es notable que los indios no han explotado nunca la planta por su fibra. La India ha desarrollado sus propias preparaciones de cannabis, que en orden ascendente de potencia son: el bhang, que es una pasta que se hace a partir de las hojas y que, agregada a otros alimentos, es comida o bebida; la ganja, que es la misma marihuana y, como esta, es fumada; y el charas, equivalente al hachís y que también se fuma. Desde la India el consumo de cannabis se extendió hacia el Oriente Cercano y África por intermedio de los árabes y los portugueses, para luego dar el salto a América (Abel, 1980, pp. 3-27; Booth, 2003, pp. 19-27; Duvall, 2015, pp. 27-49).

Los árabes conocieron las propiedades analgésicas y anestésicas de la marihuana en tiempos romanos y sus propiedades psicoactivas por el comercio con la India. El Corán prohíbe explícitamente el alcohol, pero no menciona el cannabis, lo que fue entendido por algunos como licencia para consumirlo. No obstante, muchos musulmanes, sobre todo aquellos en puestos de autoridad, rechazaron la intoxicación que produce y los únicos que lo acogieron incondicionalmente fueron los sufíes, miembros de una corriente mística que usaron la droga como una vía para la iluminación espiritual, pero que ha sido objeto de rechazo, incluso persecución, por parte de otros musulmanes. Con todo, el consumo de cannabis con fines médicos y psicoactivos se expandió junto con la fe musulmana a partir del siglo VII, y el hachís estaba bien establecido en el mundo árabe para el siglo XI. Los árabes también emplearon la planta con fines más prácticos. De los chinos aprendieron la fabricación de papel con el cáñamo como materia prima en el siglo IX y fundaron las primeras fábricas 
europeas de papel en España en el siglo XII. En el norte de África, sobre todo en Egipto y Marruecos, se desarrolló toda una cultura de sociabilidad en torno al hachís. Era ofrecido en cafés o lugares especializados, donde se consumían bebidas y dulces con cannabis o, lo más usual, se fumaba en narguiles mezclado con tabaco (Abel, 1980, pp. 36-57; Booth, 2003, pp. 34-35 y 47-52; Nahas, 1982, pp. 814-823). En Marruecos, durante el siglo XVIII la aislada región montañosa del Rif se convirtió en la principal zona de cultivo. Tradicionalmente los marroquíes han usado una pipa llamada sebsi para fumar una mezcla de cannabis y tabaco conocida como kif, que significa placer o bienestar y connota su capacidad de producir sueños y éxtasis (Bewley-Taylor, Blickman \& Jelsma, 2014, p. 12).

Comerciantes árabes difundieron el cannabis antes del año 1000 en África Oriental y desde la costa fue llevado por los propios africanos al sur y el centro del continente después del año 1500. El cultivo y consumo de cannabis adquirió gran importancia en el extremo sur de África y se convirtió en un producto significativo del comercio entre los pueblos agricultores y cazadores-recolectores antes del contacto con los europeos. Cuando los neerlandeses se establecieron en el Cabo de Buena Esperanza en 1652 encontraron que sus habitantes consumían cannabis, llamada dagga localmente, y usaron la droga, además de alcohol y tabaco, para intercambiarla con los pobladores locales por tierras, ganado y trabajo. Su consumo en grandes cantidades fuera de los contextos rituales aceleró la desintegración de los pueblos originales. Los colonos blancos cultivaron cannabis para pagar a sus trabajadores con la droga, costumbre que se mantuvo en las explotaciones mineras hasta el siglo Xx (Du Toit, 1975, pp. 81-102; Gordon, 1996, pp. 62-88). El cannabis se expandió desde el centro al occidente de África durante la era del comercio atlántico de esclavos y en su difusión participaron las redes esclavistas que se originaban en Angola. Tras la abolición de la esclavitud, los trabajadores migrantes propagaron el cannabis por la costa, desde Angola hacia Gabón y por la cuenca del río Congo. Los esclavos que fueron liberados por las armadas británica y estadounidense y llevados a África a mediados del siglo XIX introdujeron el cannabis en Sierra Leona y Liberia. Desde esta época se cultivaba cannabis en África central para abastecer a los mercados costeros y a partir de la década de 1870 


\section{8 / ANDRÉs López Restrepo}

exportadores portugueses enviaron cannabis desde Angola a diversos puntos del mundo atlántico. Hacia 1870 apareció en lo que ahora es el suroccidente de la República Democrática del Congo el movimiento político-religioso Bena Riamba, que significa "Hijos del Cannabis" y que promovía la paz y la unidad social. El cannabis era usado por sus integrantes como sacramento, medicina y, en grandes dosis, como castigo. El movimiento desapareció en la década de 1890, entre los conflictos étnicos y políticos desatados por el colonialismo belga (Duvall, 2015, pp. 93-98 y 101-103; Duvall, 2017a, 2017b, p. 236).

El cannabis fue introducido en América por las potencias coloniales, que querían usarlo para la producción de fibra. Los primeros documentos que sugieren el consumo de cannabis con fines medicinales y recreativos en la América ibérica son de mediados del siglo XVIII y corresponden a México y Brasil. La población indígena de México, con mucha experiencia en el aprovechamiento de alucinógenos de origen vegetal, se familiarizó poco a poco con la planta introducida por los españoles a mediados del siglo XVI y aprovechó y seleccionó su potencial psicoactivo. Es posible que el consumo fuese reforzado por esclavos procedentes de Angola y el Bajo Congo. En la década de 1770 el cannabis ya hacía parte de la práctica médico-religiosa mexicana y fue incorporado a las tradiciones indígenas. En la segunda mitad del siglo XIX su consumo se generalizó entre soldados y presos, y en general entre las clases bajas. A fines del siglo XIX y principios del siglo XX se propagó en México la creencia en que los consumidores de marihuana eran marginales y delincuentes, y que su uso podía generar locura y violencia (Campos, 2012, pp. 81-180; Schievenini Stefanoni, 2012, pp. 27-42). En el caso de Brasil, los esclavos trajeron de África el hábito de consumir cannabis al ser llevados a las plantaciones de azúcar creadas en el nordeste desde la segunda mitad del siglo XVI, y luego se difundió a la población indígena y a los campesinos. Los esclavos llegados a Brasil procedían de Angola, donde el cannabis estaba bien establecido - las palabras maconha o riamba y otros términos usados en Brasil para referirse al cannabis son de origen angoleño-, a diferencia de los enviados a las colonias británicas del Caribe y América del Norte, que procedían de África Occidental, donde aún no era conocida la droga. La venta de cannabis era anunciada en periódicos de Brasil en 1828 
y en la década de 1860 el explorador Richard F. Burton señaló que el cannabis crecía por todo Brasil y se había convertido en un compañero indispensable del trabajo en las plantaciones. A principios del siglo XX su presencia alcanzaba a las principales ciudades, en particular Río de Janeiro, que se abastecían de la droga producida en el nordeste, principalmente en Alagoas (Rubim de Pinho, 1975, pp. 293-196; Duvall, 2015, pp. 96, 98-101 y 107; Courtwright, 2001, p. 41).

Se ha sugerido que el uso del término marimba para designar de manera eufemística a la marihuana en Colombia podría estar relacionado con la palabra mariamba, que es el plural de cannabis en varios idiomas centroafricanos, lo cual indicaría que el cannabis fue introducido por esclavos durante el período colonial para su consumo con fines psicoactivos (Del Castillo Mathieu, 1984, p. 143). Sin embargo, no existe ningún rastro material del uso de la planta con esos fines, ni tampoco una tradición de consumo de cannabis entre los descendientes de los cimarrones, por lo que es dable desechar esta hipótesis. Durante el siglo XIX en Colombia el cannabis era utilizado en cirugía por sus propiedades analgésicas y los médicos recomendaban fumar cigarrillos de cáñamo para diversos males. Todavía a mediados del siglo XX se consumía cannabis para aliviar los dolores relacionados con la artritis (Fernández-Alonso, Galindo Bonilla \& Idrobo, 2007, p. 190). El consumo recreativo comenzó en la última década del siglo XIX, en círculos de artistas y bohemios. Su producción y uso popular son fenómenos del siglo Xx, por lo menos en lo que es su territorio actual, porque a Panamá, que hizo parte de Colombia hasta 1903, llegó a finales del siglo XIX. Existen dos teorías al respecto. Una posibilidad es que el hábito de consumir cannabis viniese de Panamá, a donde había sido llevado por trabajadores afrocaribeños que, tras la finalización de la obra del canal, en 1914, se establecieron en Colombia para laborar en las plantaciones bananeras de la United Fruit Company y en empresas petroleras. Otra teoría afirma que fue popularizado por nativos de Jamaica que llegaron a Barranquilla para trabajar como picapedreros en la década de 1920. De cualquier forma, hay coincidencia en que el cannabis fue traído por los afrocaribeños que constituyeron la corriente inmigratoria más numerosa de Colombia a fines del siglo XIX y principios del XX (Rhenals Doria \& Flórez Bolívar, 2013, 
pp. 251-252). Desde la costa Caribe, la marihuana penetró hacia el interior siguiendo la ruta del río Magdalena y durante décadas fue una droga exclusiva de sectores marginales y alguno que otro bohemio. En la década de 1920 ya existían cultivos comerciales en la Sierra Nevada de Santa Marta para satisfacer el pequeño mercado nacional (López Restrepo, 2016, pp. 38-39 y 195-196).

En Estados Unidos, el cannabis fue recomendado para fines médicos a partir de la década de 1840, pero su uso fue limitado. En las últimas décadas del siglo XIX llegó el hachís, que tuvo alguna aceptación en un momento en que el puritanismo había declarado la guerra al alcohol, pero su consumo fue muy reducido y debía hacerse en privado. Diversos testimonios indican la presencia de marihuana en Estados Unidos por primera vez en la década de 1890, la cual llegó más o menos simultáneamente por dos vías diferentes: por una parte, inmigrantes del sudoeste de Asia —árabes, turcos y armenios — cultivaron cannabis en el centro de California para su consumo y el de sus compatriotas en San Francisco; por otra parte, empezó a ser importada a través de la frontera con México para la venta a migrantes de ese país (Duvall, 2015, pp. 107-110). En estos años la prensa estadounidense reprodujo las terribles historias que circulaban en México sobre la marihuana, alimentando el temor de que la planta causase violencia y locura (Campos, 2012, pp. 203-223). Después de 1900 el comercio creció en los Estados fronterizos del sur para satisfacer la demanda de más de un millón de trabajadores de origen mexicano que llegaron en las tres primeras décadas del siglo Xx. Además, algunos marineros procedentes del Caribe comenzaron a introducirla por los puertos del golfo de México y el océano Atlántico, sobre todo Nueva Orleans, desde donde su consumo se extendió a la población afroamericana. También apareció una incipiente producción local en Estados tan lejanos de la frontera como Kansas en 1920 y hubo alguna producción de marihuana medicinal con apoyo del gobierno federal. Bien fuera cultivada o silvestre, la marihuana se encontraba fácilmente y sus precios eran bajos. En todo caso, su consumo estaba limitado a grupos minoritarios: la población negra en el sur, mexicanos en Texas y negros y mexicanos en California. En Nueva Orleans, hacia 1920 el cannabis era usado en los burdeles por prostitutas y por los músicos 
que dieron origen al jaz: quienes lo llevaron consigo al nordeste de Estados Unidos (Courtwright, 2001, pp. 41-44).

Los migrantes indios difundieron el hábito de consumir cannabis en otras posesiones británicas. Tras la abolición de la esclavitud en el Imperio británico en 1833, muchos libertos se negaron a aceptar los bajos salarios que les ofrecían las plantaciones y para remediar la falta de mano de obra las autoridades coloniales fomentaron la inmigración de trabajadores con contratos de larga duración desde China y, sobre todo, India, cuyas condiciones laborales no eran mucho mejores que las de los esclavos que les precedieron. Entre $1834 \mathrm{y}$ 1938, cinco y medio millones de indios emigraron como trabajadores a doce colonias diferentes, entre ellas Natal, Mauricio, Australia, Fiyi, Jamaica, Trinidad y la Guayana británica, la actual Guyana, donde llevaron la costumbre de consumir cannabis. En el caso de Natal, en la actual Sudáfrica, los indios llegaron a partir de 1860 para laborar en las plantaciones de azúcar y se encontraron con que ya existía una tradición de cultivo y consumo de cannabis (Mills, 2007, pp. 168-172; Duvall, 2015, pp. 101-105). Al Caribe llegaron entre 1838 y 1917 casi medio millón de trabajadores indios adeudados a trabajar en las plantaciones de azúcar de Jamaica, Trinidad y Guyana, quienes cultivaron y consumieron la planta. Debido al vínculo del cannabis con la India, el Caribe anglófono adoptó la palabra hindi ganja para designar la droga. En Guyana y Trinidad el cannabis se mantuvo en el ámbito de la población india hasta la década de 1960; en Jamaica, en cambio, su consumo se extendió entre la población negra trabajadora rural y urbana, y se convirtió en parte esencial de los ritos del movimiento político-religioso rastafari, nacido en la década de 1930 a partir de la amalgamación de creencias surasiáticas y centroafricanas en un contexto caribeño. Desde fines del siglo XIX el cannabis se expandió desde las islas del Caribe británico hacia los países hispanoamericanos costeros, ya que fue llevada por las decenas de miles de afrocaribeños que llegaron a trabajar en el canal de Panamá y en las plantaciones de banano en Costa Rica y Panamá y de azúcar en Cuba (Mills, 2003, p. 13; Angrosino, 2003, pp. 101-116; Comitas, 1975, pp. 119-132; Abel, 1980, pp. 101-102; Courtwright, 2001, pp. 41-42). 
Para los europeos, el cannabis fue ante todo una fibra. Con ese fin la explotaron griegos y romanos, y durante la Edad Media adquirió gran importancia en la industria naviera, como materia prima del velamen y el cordaje de los barcos. Las grandes potencias marítimas europeas de la Edad Moderna impulsaron el cultivo de cáñamo, con más reveses que éxitos, en las metrópolis y en sus colonias, en particular las americanas. Debido a que la demanda superaba a la producción, se vieron obligadas a importar la fibra desde Rusia, que se convirtió en su principal productor y exportador mundial (Borougerdi, 2014, pp. 31-40 y 69-134; Abel, 1980, pp. 28-35 y 61-75; Booth, 2003, pp. 27-42; Duvall, 2015, pp. 49-83). Los europeos también usaron ocasionalmente el cannabis en algunas preparaciones medicinales. Sus efectos psicoactivos no parecen haber sido conocidos antes del siglo XIX. Se ha afirmado que algunos sectores populares sí experimentaron con anterioridad esos efectos y que el cannabis era una de las drogas usadas con fines alucinógenos en las prácticas de la brujería, pero en todo caso este tipo de consumo no dejó una huella significativa en la sociedad (Abel, 1980, pp. 106-110). El cannabis empezó a aparecer en las farmacopeas europeas en el siglo XVIII, donde se recomendaba su uso con fines antibióticos, analgésicos y antiinflamatorios. Gracias a sus aventuras coloniales, los europeos descubrieron, o redescubrieron, su potencial psicoactivo. Tras la ocupación de Egipto en 1798, los franceses conocieron el hachís y al regresar a Europa luego de su derrota en 1801 llevaron el gusto por la droga y empezaron a importarla del Medio Oriente. Más tarde el hachís fue adoptado en círculos intelectuales, sobre todo de escritores, que buscaban mediante las drogas estimular su imaginación y encontrar la fuente de sus emociones. En la década de 1840 operó el Club de Hachís, un grupo de artistas parisinos reunidos en torno al escritor Théophile Gautier y el médico Jacques-Joseph Moreau, quien usó el hachís para realizar el primer trabajo sistemático sobre los efectos de las drogas en el sistema nervioso central (Abel, 1980, pp. 119-121 y 148-167; Booth, 2003, pp. 79-92). Durante mucho tiempo el cannabis fue una droga de marginados y el único lugar de Europa donde su consumo tuvo importancia fue en Grecia, donde se difundió después de la Primera Guerra Mundial tras la llegada desde Asia Menor de las tropas desmovilizadas y de un millón de repatriados (Abel, 1980, 
pp. 134-135; Booth, 2003, pp. 120-134 y 151-152; Stefanis, Ballas \& Madianou, 1975, pp. 310-315).

Un médico británico, William Brooke O’Shaughnessy, quien conoció el cannabis en la India y experimentó con la planta en animales y humanos, hizo el primer estudio científico de sus propiedades terapéuticas. En varias publicaciones a partir de 1839, O’Shaughnessy recomendó su uso como narcótico, analgésico, estimulante y antiepiléptico, entre otras aplicaciones, y llevó la droga a Europa. Como resultado de su trabajo, desde 1840 se empezaron a comerciar tinturas de cannabis, que eran soluciones de la droga en alcohol para ser ingeridas oralmente. El cannabis también hizo parte de medicinas patentadas promovidas para diversas condiciones médicas y se vendía como calmante para el dolor en pastillas cubiertas de azúcar para impedir que se pegasen entre sí (Booth, 2003, pp. 109-119; Mills, 2003, pp. 39-46; Borougerdi, 2014, pp. 135-153). El mercado internacional de preparaciones farmacéuticas de cannabis se mantuvo hasta 1940 y en algunos lugares fueron recetadas hasta la década de 1960. Los cigarettes indiennes eran cigarrillos que contenían cannabis, belladona, datura, beleño negro y opio, y fueron producidos por la farmacéutica francesa Grimault, que recomendaba su uso contra el asma, la tos, el insomnio y la amnesia, y los exportó a todo el mundo entre las décadas de 1860 y 1920. No obstante, había evidentes limitaciones al uso del cannabis como medicina. Quienes experimentaron con la droga advirtieron desde un principio algunos efectos secundarios, como alucinaciones o paranoia. A partir de estudios realizados en la India y Egipto, se extendió la idea de que el cannabis era causante de crimen y locura. Se planteó que la droga era adictiva, incluso más peligrosa que el opio, y en 1894 el médico británico Norman Kerr incluyó la cannabinomanía en su libro sobre narcomanía. Y su ingestión era complicada. El cannabis no es soluble en agua, por lo que no puede ser inyectado, y apenas en 1964 se pudo aislar y determinar la estructura de su principio activo, el THC. Para principios del siglo Xx, la ciencia médica había perdido el interés por la planta y se le prescribía muy raramente (Duvall, 2015, pp. 135-139; Snelders, Kaplan \& Pieters, 2006, pp. 95-114). 


\section{Las naciones contra el cannabis}

Las sociedades que primero prohibieron el cannabis se concentran en el mundo musulmán, África subsahariana y América Latina y el Caribe. En la India, el proceso de regulación también fue temprano. Donde primero se observó esa voluntad de acabar con los placeres del cannabis fue en el mundo musulmán. Ya se mencionó que el Corán prohíbe explícitamente el alcohol, pero no dice nada sobre el cannabis. Algunos entendieron que la prohibición del alcohol se extendía a todas las sustancias psicoactivas, otros defendieron que el cannabis estaba autorizado de manera tácita; entre los musulmanes que rechazaban la droga estaban muchas autoridades religiosas y civiles. En Egipto hubo prohibiciones esporádicas del hachís en los siglos XIII y XIV, pero su consumo y producción persistieron (Nahas, 1982, pp. 814-831). El uso de cannabis en el mundo musulmán tendía a ser asociado con la población masculina de las clases bajas, mientras que la élite, pese al tabú religioso, prefería el alcohol, que en todo caso era mucho más costoso. Siglos después, durante la ocupación de Egipto, los franceses se sorprendieron por la extensión del consumo de hachís entre la población local y adoptaron la costumbre, dado que les era imposible conseguir alcohol por la prohibición islámica. Los oficiales al mando de la tropa reportaron que el consumo de hachís hacía que las tropas se relajasen y cometiesen actos de indisciplina, por lo que en octubre de 1800 el comandante de la fuerza expedicionaria, el general Jacques-François de Menou, prohibió la producción, distribución y consumo de la droga en todo Egipto. Los franceses fueron derrotados en 1801 y el nuevo gobierno derogó la prohibición (Duvall, 2017a; Booth, 2003, pp. 47-52).

En la segunda mitad del siglo XIX, los líderes nacionales egipcios emprendieron un vigoroso proceso de modernización de tipo occidental y llegaron a ver en el consumo de hachís un obstáculo para su proyecto. Por esta razón, en 1868 Egipto prohibió la producción, importación y consumo de hachís. En 1874 se permitió su importación sujeta al pago de un impuesto, pero en 1879 se ratificó la prohibición del cultivo e importación de la droga y se ordenó la destrucción de la confiscada por las aduanas (Abel, 1980, pp. 133-134). Al año siguiente un decreto 
ordenó la destrucción de los cultivos, bajo pena de multa, y en 1881 se hizo responsables a los jefes de las aldeas por el cannabis producido en sus jurisdicciones. Dada la cantidad limitada de tierra cultivable, la prohibición de la producción fue relativamente eficaz y existen registros oficiales de erradicación de plantas en la década de 1880 . Sin embargo, la prohibición del consumo no fue tan efectiva, puesto que el contrabando se encargó de atender la demanda. Al hacerse al control de Egipto en 1882, los británicos conservaron la política de prohibición del cannabis, la cual se mantuvo luego de que el país alcanzara su independencia en 1922. Debido a la persistencia del contrabando, Egipto concluyó que requería la ayuda de las demás naciones para detener el flujo ilegal de la droga y sería el país que más insistió por incluir al cannabis en el régimen internacional de control de drogas (Kozma, 2011, pp. 444-455). El interés de Egipto por controlar el cannabis derivaba en parte de la creencia en que el cannabis producía locura. A partir de 1896 el británico John Warnock, quien sirvió durante casi tres décadas como director médico del Hospital para Dementes de El Cairo, publicó una serie de trabajos en que sostenía que el hachís era la causa de una buena parte de la locura y el crimen en Egipto. Los diagnósticos de Warnock son sospechosos, dado que nunca se tomó la molestia de aprender árabe y dependió de intérpretes para comunicarse con sus pacientes y el personal del hospital. No obstante, su trabajo tendría mucho impacto sobre la política de Egipto frente a la droga y fue citado en los reportes que el país presentó a la Sociedad de Naciones en la década de 1920 (Mills, 2003, pp. 177-187).

Las restricciones al cannabis en el África subsahariana se remontan a mediados del siglo XIX. El Madagascar precolonial prohibió el cannabis en 1870, pero fueron las potencias coloniales las más activas en esta materia. Argumentaban que la planta era nociva para la salud, pero en realidad estaban más preocupadas por el control de la mano de obra. Dado que en las sociedades coloniales esa mano de obra correspondía a grupos étnicos considerados inferiores, la legislación contra el cannabis tuvo un carácter claramente racista. En Angola, una ley de 1913 prohibió el consumo de cannabis por parte de las tropas coloniales y promovió la sustitución de los cultivos por tabaco. Con excepción 
de Marruecos y Túnez, la droga había sido declarada ilegal en todo el continente para la década de 1910. La prohibición impuesta por los regímenes coloniales se mantuvo después de la independencia, como parte de los compromisos internacionales de las nuevas naciones (Duvall, 2017a). Mención especial requieren Sudáfrica y las colonias que la precedieron. Desde fines del siglo XIX hubo inquietud entre la población blanca por el consumo de dagga entre los mestizos y la población de origen indio, así como por sus supuestos efectos: la mala salud, el crimen y la indolencia de los trabajadores. En 1870 Natal aprobó una ley que prohibía a los indios el consumo y posesión de cannabis, en 1891 la Colonia del Cabo prohibió la droga —aunque la ley no fue aplicada hasta 1912, en el contexto del aumento de la tensión racial que ocurrió hacia principios de siglo-, y lo mismo hizo el Estado Libre de Orange en 1903. La cuarta colonia, Transvaal, estuvo en desacuerdo con la prohibición, pues su principal actividad era la minería de oro y los empresarios mineros no querían cortar el suministro de la droga a sus trabajadores negros e indios. Finalmente, pese a la oposición de Transvaal, en junio de 1922 el gobierno blanco minoritario de Sudáfrica prohibió la producción, comercio, posesión y consumo de opiáceos, cocaína y cannabis. $\mathrm{Al}$ año siguiente, Sudáfrica pidió a la Sociedad de Naciones la inclusión del cannabis dentro del régimen de control, por ser "la más importante de todas las drogas creadoras de hábito” (Paterson, 2009, pp. 1-55; Mills, 2007, pp. $165-$ 172; Booth, 2003, pp. 140-142; Chanock, 2001, pp. 92-96).

En América Latina las regulaciones del cannabis también respondieron a preocupaciones raciales y socioeconómicas. El primer país donde se prohibió la droga fue Brasil, donde en 1830 el concejo municipal de Río de Janeiro, en ese entonces la capital, proscribió su venta y consumo, así como su presencia en sitios públicos. Otras ciudades brasileras hicieron lo mismo en las décadas siguientes, aunque no hay evidencia de que estas normas hayan sido aplicadas. Finalmente, la legislación federal clasificó al cannabis como un narcótico en 1932 y prohibió su consumo y venta en 1938 (Bewley-Taylor et al., 2014, p. 10). México también tiene una vieja historia de prohibición del cannabis. En un principio, las regulaciones que afectaron la planta se sustentaban en el deseo de restringir el acceso a ciertas sustancias 
peligrosas, por lo que ponían al cannabis en la misma categoría que el opio, la belladona y la datura. Con ese fin la ciudad de México prohibió en 1869 la venta de marihuana y luego le siguieron otras ciudades y Estados. En 1891, el primer Código Sanitario Federal instauró que los extractos y tinturas del cannabis debían ser expendidos mediante receta y en cantidades limitadas, y que la planta debía ser vendida a las farmacias por las herbolarias que la recogían o cultivaban. En marzo de 1920 el Departamento de Salubridad Pública, en un decreto que se proponía impedir la degeneración de la raza, expidió un decreto que prohibió el cultivo y comercio de cannabis, incluso para fines industriales, bajo pena de decomiso de la droga y multa (Campos, 2012, pp. 181-201; Schievenini Stefanoni, 2012, pp. 47-54 y 59-62). En Colombia, la primera norma sobre las drogas, la Ley 11 de 1920, recogía en líneas generales lo acordado en la Convención Internacional del Opio de 1912 y en concreto consideraba, como la Convención, que los únicos usos aceptables para las "drogas formadoras de hábito pernicioso" eran los médicos y legítimos. Pero, a diferencia de la Convención, que limitaba sus alcances a los opiáceos y la cocaína, la Ley 11 incluyó al C. indica, por lo que esta droga debía ser comprada en farmacia o botica y con receta médica. Posteriormente, un decreto de agosto de 1930 prohibió la importación y venta de marihuana, pero permitía la importación bajo licencia del cáñamo indio para su uso como fibra. La política de represión al cannabis culminó con una resolución de septiembre de 1939, la cual prohibió el cultivo de la marihuana y concedió 60 días para destruir las plantaciones existentes (López Restrepo, 2016, pp. 143-147, 157-159 y 173-174).

Los países anteriores prohibieron el cannabis; otros optaron por regular y lucrarse de la droga, al menos por un tiempo. Fue el caso del Caribe anglófono, donde desde mediados del siglo XIX las autoridades coloniales y religiosas y los dueños de las plantaciones vieron con malos ojos el consumo de la droga por parte de la población de origen indio. Esta actitud se basaba en la creencia, propia de la ideología de la supremacía blanca, de que el consumo tenía como resultado el desinterés por el trabajo y la violencia hacia las mujeres. Ciertamente había muchos casos de violencia de género, pero estaba más relacionada con el hecho de que llegaban más hombres que 
mujeres desde la India. Las administraciones coloniales trataron de desincentivar el consumo de cannabis estableciendo el pago de licencias para su cultivo y venta, lo que estimuló el mercado negro. En Guyana, las regulaciones se remontan a 1861 y su sistema de licencias para la venta al detal era manejado por comerciantes chinos, que al parecer recibían el cannabis de la India vía Londres. Trinidad aprobó en 1885 una ordenanza que elevó los impuestos sobre el cannabis y constituyó un sistema de licencias para su venta, e incluso prohibió la droga entre 1894 y 1899. Aunque el cannabis no fue incluido en la Convención Internacional del Opio de 1912, su aprobación provocó el endurecimiento de las normas sobre la planta en el Caribe anglófono. Así, Guyana en 1913 y Trinidad en 1915 prohibieron el cultivo de la planta, mientras que Jamaica, que hasta el momento no la había regulado, prohibió en 1913 tanto el cultivo como la importación de cannabis. En respuesta a la Convención del Opio de 1925 y a la Ley de Drogas Peligrosas del Reino Unido de ese mismo año, las posesiones del Caribe británico declararon ilegal todo lo relacionado con el cannabis entre 1928 y 1938. En 1928 Guyana y Trinidad prohibieron el consumo de cannabis y dieron fin al sistema de licencias para su venta (Fraser, 1974, pp. 361-385; Angrosino, 2003, pp. 101-116).

Un proceso similar ocurrió en el Magreb. Los sultanes de Marruecos cobraban impuestos sobre el kif, que eran recaudados por las administraciones locales. En 1890 se determinó que solo se podía cultivar cannabis en cinco aldeas de la región montañosa del Rif. Poco después, en 1906, se otorgó el monopolio sobre el kif a una multinacional de capital francés, la cual autorizaba el cultivo de cannabis y tabaco a los campesinos, y a cambio estos debían entregar sus cosechas en las factorías de la empresa en Tánger y Casablanca, donde se producía kif con dos partes de cannabis y una de tabaco. Sin embargo, muchos campesinos cultivaban cannabis pese a carecer de licencia. Marruecos perdió su independencia en 1912 y fue dividido en dos áreas de influencia, francesa y española —el Rif quedó en la zona española-, y las dos potencias coloniales mantuvieron los términos del monopolio de kif. Durante la revuelta anticolonial del Rif, ocurrida entre 1921 y 1926, su líder, Abd-el-Krim, proscribió la producción y consumo de kif por considerarlo contrario al islam. Tras 
el fin de la revuelta, los monopolios fueron restablecidos. En 1954, Francia prohibió el cultivo de cannabis en su protectorado. Cuando Marruecos recuperó su independencia en 1956, unificando los protectorados francés y español, la prohibición del cannabis se extendió a la antigua zona española. Esto, junto con otros factores, provocó una rebelión en el Rif en 1958, que fue duramente reprimida. Para disminuir las tensiones, a partir de entonces el gobierno marroquí ha tolerado el cultivo de cannabis en las cinco aldeas históricas. En Túnez, los otomanos también establecieron en 1870 un monopolio sobre el cultivo y la venta de cannabis, conocido en el país como takrouri, que los franceses mantuvieron al hacerse con el control del país en 1881, pero que desmontaron, al igual que en Marruecos, al prohibir el cannabis en 1954 (Blickman, 2017, pp. 3-6; Bewley-Taylor et al., 2014, p. 12).

La India ha sido el país que más se ha resistido a la prohibición del cannabis. Los británicos rechazaban su consumo, pero optaron por lucrarse de su comercio mediante una política de impuestos que buscaba a la vez desincentivar su uso y obtener ingresos fiscales. Así, a partir de 1798 la Compañía Británica de las Indias Orientales requirió a los cultivadores y comerciantes de la droga el pago de una licencia para ejercer su actividad. En la segunda mitad del siglo XIX, las autoridades coloniales británicas de la India vieron el cannabis con creciente preocupación al generalizarse la idea de que su uso excesivo era el principal culpable de los casos de locura y de la violencia y el desorden provocados por quienes la padecían. Después de estudiar el problema, el gobierno colonial concluyó en 1873 que la planta no incitaba al crimen más que otras drogas, incluido el alcohol, pero sí podía producir locura en cantidades excesivas. Debido a su utilidad como medicina y a que el número de casos de locura causados por su consumo era bajo, el gobierno decidió mantener sin cambios su política frente al cannabis. Aunque menos significativos que los provenientes del opio, los recursos generados por el cannabis no eran despreciables: a fines del XIX generaba una décima parte de los impuestos al consumo y en Bengala llegaba a la quinta parte. El debate sobre los efectos del cannabis continuó, por lo que el gobierno colonial promovió la creación de la Comisión de Drogas de Cáñamo 
Indio, que, tras un poco más de un año de trabajo, entregó en 1895 un extenso informe de siete volúmenes que sigue siendo, pese a sus sesgos, el conjunto de información sobre el cannabis más detallado que existe. La Comisión descartó la causalidad entre el consumo de cannabis y el crimen, y sí consideró que podía inducir locura, pero solo en quienes la usaban de manera excesiva y tenían alguna debilidad o disposición hereditaria. La Comisión concluyó que la droga no era inofensiva, pero sus beneficios superaban a sus daños, por lo que su prohibición no era "ni necesaria ni conveniente". Y para evitar el consumo excesivo bastaba una política de "control y restricción" mediante impuestos elevados, licencias para el cultivo y comercio, y límites a la cantidad que podía poseerse (Mills, 2003, pp. 47-51 y 82-140; Shamir \& Hacker, 2001, pp. 438-461).

Es notable que los británicos adoptaran políticas tan diferentes frente al cannabis en sus distintos dominios. Aunque desaprobaban su consumo, su política dependió de qué tipo de relación querían establecer con sus sujetos en cada colonia. La preferencia del gobierno era lucrarse del cannabis, pero en aquellos lugares en que ya existía una política bien definida frente a la droga prefirieron respetarla. La India y Egipto ya tenían unas normas cuando llegaron los británicos y estos se encargaron de administrar esa tradición heredada, alterándola solo en aspectos secundarios. En la India mantuvieron y perfeccionaron el sistema de licencias e impuestos recibido de los mogoles, lo cual les permitió gravar y lucrarse de la droga, al igual que hicieron con el opio. En Egipto mantuvieron la prohibición instituida durante el Jedivato, pese a que los británicos tenían dudas sobre la conveniencia de proscribir una droga que se daba de manera silvestre y consideraban que la prohibición creaba resistencias, incentivaba el contrabando y la corrupción y reducía los ingresos fiscales. Por todas estas razones algunos funcionarios británicos llegaron a proponer que Egipto adoptase una política para el cannabis similar a la de India (Kozma, 2011, pp. 448-449 y 451). Correspondería a los gobiernos soberanos de la India y Egipto modificar la política legada por los británicos. En el Caribe, donde su control era mayor, los británicos no tuvieron que hacer concesiones a las normas locales e impusieron la política de su preferencia. Lo que preferían era el modelo de la India y de 
esta manera establecieron un sistema de licencias que les proporcionaba recursos y al mismo tiempo limitaba el consumo de una droga que rechazaban. Esta situación se mantuvo hasta la entrada en vigor del régimen internacional de control de drogas, que los llevó a prohibir el cannabis. Sudáfrica fue un caso aparte, ya que por temor a la población nativa la minoría blanca apoyó regulaciones que fortalecieron su proyecto político supremacista.

\section{La comunidad internacional contra el cannabis}

El régimen internacional de drogas que empezó a tomar forma a principios del siglo XX no prestó mucho interés inicialmente al cannabis. Después de todo, la construcción de ese régimen era cosa de los países centrales y los que se preocupaban por el tema del cannabis eran sobre todo países secundarios. La primera vez que la droga se mencionó en un escenario internacional fue cuando se estaba discutiendo la invitación que hizo Estados Unidos para una Conferencia Internacional del Opio que diese seguimiento a las iniciativas planteadas en la Comisión Internacional del Opio, que se había reunido en Shanghái en 1909. Fue por iniciativa de Italia, que, viendo con preocupación cómo su territorio se había convertido en lugar de paso para el contrabando de hachís, a principios de 1910 condicionó su participación en la Conferencia a que esta se ocupase del control a la producción y el comercio de cannabis. Se ha afirmado que el interés de Italia se debía al problema del hachís que encontró en sus nuevas colonias de Tripolitania y Cirenaica, en la actual Libia. Pero Italia se hizo a esas colonias como resultado de su guerra con el Imperio otomano, que ocurrió entre 1911 y 1912, después de pedir que la Conferencia considerase el asunto del cannabis. Esta confusión se originó en un apartado del libro de Lowes, quien, luego de decir que Italia había pedido en febrero de 1910 que la Conferencia tratase el control del cannabis, anotaba que en noviembre de 1911 había proclamado un protectorado sobre Tripolitania y Cirenaica, donde existía un problema con el hachís. Lowes (1966, p. 173) se limitaba a señalar una coincidencia interesante, pero no afirmaba que la preocupación de 
Italia era por la situación en sus nuevas colonias, como se malinterpretó después. La Conferencia se reunió en La Haya entre diciembre de 1911 y enero de 1912 y acordó la Convención Internacional del Opio, el primer instrumento internacional con miras al control de drogas. La delegación italiana no presentó ninguna propuesta sobre el cannabis ni sobre ningún otro asunto y solo asistió al primer día de la Conferencia, que no se ocupó del cannabis ni lo mencionó en la Convención. Por sugerencia de Estados Unidos, el Protocolo Final de la Conferencia manifestó la conveniencia de que los países participantes estudiasen la droga para en un futuro determinar si era necesario regular su abuso mediante legislación interna o acuerdo internacional (López Restrepo, 2016, pp. 84-85; Mills, 2003, pp. 154-156; Taylor, 1969, pp. 87-88; Musto, 1999, p. 51).

La Sociedad de Naciones, nacida de las cenizas de la Primera Guerra Mundial, se encargó del régimen de control de drogas. La primera vez que el cannabis fue considerado por la Sociedad fue en 1923, cuando el gobierno de Sudáfrica manifestó en una comunicación dirigida al Comité del Opio de la Sociedad que consideraba al cannabis como la peor de las drogas formadoras de hábito y pidió que se incluyese en la Convención de 1912. Finalmente, el cannabis fue incorporado al régimen durante la Segunda Conferencia Internacional de Opio, que se reunió en Ginebra entre noviembre de 1924 y febrero de 1925. En la Conferencia, Egipto planteó que el hachís era muy adictivo, tan nocivo, si no más, que el opio, y la principal causa de locura en su territorio, por lo que pedía que el uso del cannabis fuese restringido a "propósitos médicos y científicos". La India, con apoyo del Reino Unido y Francia, rechazó la propuesta egipcia y defendió el uso tradicional de la droga. La nueva Convención Internacional de Opio, que resultó de la Conferencia y que reemplazó e hizo más estricta la Convención de 1912, incorporó el cannabis, según lo pedía Egipto, pero, debido a la oposición india, lo hizo bajo un régimen más laxo que el de las demás drogas. La Convención determinó que el cannabis y las drogas manufacturadas que lo incluyeran en su composición solo podían ser producidas, comerciadas y usadas con fines médicos y científicos; no obstante, dado que la Convención no determinó qué quería decir con fines médicos y científicos, esta provisión no tuvo 
ningún efecto. Y a diferencia de las otras drogas, no se estableció la obligación de proporcionar estimados de su producción a la Junta Central Permanente del Opio, creada por la misma Convención para supervisar el comercio internacional lícito de drogas. Varios delegados admitieron que nunca habían oído del cannabis, pero votaron a favor de someterlo a control porque fueron convencidos por los egipcios o quisieron apoyarlos en un tema que no generaba resistencia en sus países (Willoughby, 1925, pp. 374-384; Mills, 2003, pp. 160-177; López Restrepo, 2016, pp. 103-106).

A partir de entonces la dinámica de control tomó impulso propio. Los Estados que aún no tenían legislación sobre el cannabis aprobaron leyes destinadas a prohibir su posesión y consumo, incluso aquellos en que la droga no se usaba o no constituía un problema. En muchos lugares las leyes nacionales excedieron las obligaciones de la Convención. Sin embargo, Estados Unidos, que desde un principio lideró el establecimiento del régimen internacional de control, se resistió durante mucho tiempo a aprobar una ley contra el cannabis, pues consideraba que su regulación debía estar en manos de los Estados y no ameritaba la intervención del gobierno federal. Este debía concentrarse en la represión de la heroína, una sustancia mucho más peligrosa, y no distraerse con una planta que crecía libremente en muchas partes del país. A partir de 1914 casi todos los Estados aprobaron legislación anticannábica, cuya justificación en muchos casos incluía el prejuicio contra los mexicanos. El rechazo a los mexicanos aumentó durante la crisis económica de los años treinta, pues se consideró que competían por los trabajos de los blancos, y los Estados fronterizos del sur, donde se concentraban los migrantes mexicanos, demandaron una ley nacional contra el cannabis, argumentando que su consumo estimulaba el crimen. Finalmente, hacia 1935 las presiones de esos Estados obligaron al gobierno federal a apoyar una ley nacional que fue promovida mediante una campaña racista, xenofóbica y colmada de exageraciones y mentiras sobre los supuestos estragos causados por el cannabis. En octubre de 1937 fue aprobada la Ley de Imposición de la Marihuana, la cual instituyó un impuesto sobre todos los participantes en la producción y comercio del cannabis y sobre todas sus transacciones. No obstante, el gobierno no distribuyó los timbres que 
indicaban el pago del impuesto, por lo que en adelante el comercio legal de la droga fue imposible: la Ley de la Marihuana se presentaba como una medida tributaria, pero su propósito real era la prohibición de la droga (Musto, 1999, pp. 216-229; Bonnie \& Whitebread II, 1999, pp. 32-174; Duvall, 2015, p. 109).

Una de las principales consecuencias de la Segunda Guerra Mundial fue la conversión de Estados Unidos en una potencia hegemónica, lo que aprovechó para impulsar un régimen de control de drogas aún más restrictivo. La Organización de las Naciones Unidas quiso consolidar los diversos tratados que conformaban ese régimen y, tras un proceso que se inició en 1948, se acordó en 1961 la Convención Única de Estupefacientes, que aún sirve de fundamento del régimen. Esta Convención incluyó el cannabis y la hoja de coca en el grupo de sustancias más adictivas y nocivas, determinó que su valor terapéutico era muy reducido y limitó su producción, comercio, consumo y posesión "exclusivamente" a fines médicos y científicos, lo que significó prohibir su uso tradicional. Estas disposiciones sobre el cannabis se referían únicamente a los cogollos y a la resina, pues la India consiguió que las hojas, que son la materia prima del bhang, no quedaran sometidas a control, aunque las partes se obligaron a impedir su "uso indebido o tráfico ilícito". También quedaba exento de control el cultivo de la planta con "fines industriales (fibras y semillas) u hortícolas". Para hacer más tolerable el impacto de las medidas, la Convención permitió que los Estados autorizaran el uso para fines no médicos y no científicos del cannabis y de la coca por un período de hasta 25 años luego de su entrada en vigor, al cabo del cual las plantas quedarían totalmente prohibidas. Este plazo se cumplió el 13 de diciembre de 1989 y desde entonces toda la producción y uso del cannabis que no tenga fines médicos o científicos están prohibidos por el régimen internacional (Mills, 2013, pp. 95-115; Bewley-Taylor et al., 2014, pp. 18, 20-25).

\section{Conclusiones}

La prohibición del cannabis fue resultado de un proceso que se dio en dos etapas. En la primera, hasta 1925, intervinieron tres países, 
Egipto, Sudáfrica y la India —Egipto y Sudáfrica a favor de la prohibición, la India en contra—, que tenían en común su carácter secundario en el escenario mundial y su posición subordinada respecto al Reino Unido. El hecho de que estos países influyeran en el estatus del cannabis se debió a dos factores principales: la poca importancia que tenía entonces la droga, tanto que el Reino Unido, aunque apoyó a la India, no ejerció una presión significativa para eximir al cannabis del régimen de control; y la creación de la Sociedad de Naciones, que por primera vez permitió que todos los países del mundo participaran en un foro internacional en condiciones de igualdad. En la segunda etapa, después de la Segunda Guerra Mundial, Estados Unidos fue determinante para la prohibición del consumo de cannabis con fines psicoactivos. El cannabis, que era una droga de marginales o de habitantes de países subalternos, fue prohibido definitivamente en 1961. $\mathrm{O}$ al menos parecía, dado que las cosas cambiaron muy pronto. No estaba aún seca la tinta con la que se había firmado la Convención Única cuando el uso del cannabis se generalizó entre la juventud rebelde, primero de Estados Unidos, el país abanderado de la eliminación de la droga, y luego, progresivamente, entre las clases medias del resto del planeta. Hubo quienes profetizaron que el auge del cannabis era un capricho pasajero y que las cosas eventualmente se enderezarían, pero este fenómeno, que comenzó en la década de 1960, aún no da indicios de aflojar. Más recientemente ha comenzado a extenderse la aceptación del consumo de cannabis con fines recreativos y pareciera que la planta ha emprendido el camino, al parecer imparable, hacia su plena legalización.

La despenalización o legalización tiene varios argumentos a favor. Estudios clínicos recientes reportan que el cannabis es eficaz para diversas condiciones como el dolor crónico y las náuseas y el vómito causados por la quimioterapia y estimula el apetito en pacientes con cáncer o sida. La legalización con fines médicos del cannabis ha facilitado la investigación de sus usos terapéuticos. Por otra parte, están las razones ecológicas. Los cultivos ilegales de cannabis son mucho más nocivos para el medio ambiente que los legales, pues por hacerse en espacios cerrados hacen un uso intensivo de electricidad y fertilizantes. La desaparición del mercado negro también acabaría con la 
corrupción y la violencia asociadas. Además, las drogas del mercado negro tienen muchos problemas, desde niveles de THC indeterminados hasta su mezcla con venenos y otras sustancias. También hay argumentos económicos, ya que disminuirían los costos de la represión y el Estado podría obtener ingresos de la producción legal de cannabis.

Claro está que persisten varios obstáculos para el cambio. En primer lugar, el uso recreativo del cannabis es contrario a lo dispuesto por la Convención de 1961. Si los países no quieren incumplir con sus obligaciones internacionales, tienen dos opciones: retirarse de la Convención o cambiarla. Ambas alternativas son costosas y no es claro en qué sentido van a decantarse los países que están legalizando el cannabis. Pero sobre todo hay que reconocer que el cannabis no es una droga inocua, como parecen creer algunos de sus promotores; entre sus efectos están la dependencia y se la asocia con la psicosis. La despenalización o la legalización pueden provocar un aumento del consumo y, por lo tanto, de los riesgos asociados. Por ende, es necesario establecer políticas fundadas en el mejor conocimiento disponible de los beneficios y daños de la planta y de las consecuencias de las distintas formas de regulación.

\section{Referencias}

Abel, E. L. (1980). Maribuana: the first twelve thousand years. New York-London: Plenum Press.

Angrosino, M. V. (2003). Rum and ganja: indenture, drug foods, labor motivation, and the evolution of the modern sugar industry in Trinidad. En W. Jankowiak \& D. Bradburd (Eds.), Drugs, labor, and colonial expansion (pp. 101-116). Tucson: The University of Arizona Press.

Bewley-Taylor, D., Blickman, T., \& Jelsma, M. (2014). Auge y caída de la probibición del cannabis. La historia del cannabis en el sistema de control de drogas de la ONU y opciones de reforma. Amsterdam-Swansea: Transnational Institute-Global Drug Policy Observatory.

Blickman, T. (2017). Marruecos y el cannabis. Reducción, contención o aceptación. Amsterdam: Transnational Institute. 
Bonnie, R. J., \& Whitebread II, C. H. (1999). The marijuana conviction: a history of marijuana probibition in the United States ( $2^{\text {th }}$ ed.). NewYork: The Lindesmith Center.

Booth, M. (2003). Cannabis. A bistory. New York: Picador.

Borougerdi, B. J. (2014). Cord of empire, exotic intoxicant: hemp and culture in the Atlantic world, 1600-1900 (Tesis de PhD, Universidad de Texas en Arlington, Arlington, Estados Unidos).

Campos, I. (2012). Home grown: marijuana and the origins of Mexico's war on drugs. Chapell Hill: The University of North Carolina Press.

Chanock, M. (2001). The making of South African legal culture, 1902-1936: fear, favour and prejudice. Cambridge: Cambridge University Press.

Comitas, L. (1975). The social nexus of ganja in Jamaica. En V. Rubin (Ed.), Cannabis and culture (pp. 119-132). La Haya-Paris: Mouton Publishers.

Courtwright, D. T. (2001). Forces of habit: drugs and the making of the modern world. Cambridge-London: Harvard University Press.

Del Castillo Mathieu, N. (1984). El léxico negro-africano de San Basilio de Palenque. Thesaurus. Boletin del Instituto Caro y Cuervo, 39(1-3), 80-169.

Du Toit, B. M. (1975). Dagga: the history and ethnographic setting of Cannabis sativa in Southern Africa. En V. Rubin (Ed.), Cannabis and culture (pp. 81-116). La Haya-Paris: Mouton Publishers.

Duvall, C. (2015). Cannabis. London: Reaktion Books.

Duvall, C. S. (2017a). Cannabis and tobacco in precolonial and colonial Africa. In Oxford research encyclopedia of African history. https://doi. org/10.193/acrefore/9780190277734.013.44

Duvall, C. S. (2017b). Science, society, and knowledge of the Columbian exchange: the case of Cannabis. En E. Vaz, C. Joanaz de Melo \& L. M. Costa Pinto (Eds.), Environmental history in the making. Vol. I. Explaining (pp. 225-241). Basilea: Springer.

Escohotado, A. (1997). La cuestión del cáñamo. Una propuesta constructiva sobre hachis y maribuana. Barcelona: Anagrama.

Fernández-Alonso, J. L., Galindo Bonilla, A., \& Idrobo, J. M. (2007). Las plantas como evidencia legal. Desarrollo de la botánica forense en Colombia. Revista de la Academia Colombiana de Ciencias Exactas, Físicas y Naturales, 31(119), 181-198.

Fraser, H. A. (1974). The law and cannabis in the West Indies. Social and Economic Studies, 23(3): 361-385. Recuperado de http://www.jstor. org/stable/27861515 
Gordon, D. (1996). From rituals of rapture to dependence: the political economy of Khoikhoi narcotic consumption, c. 14871870. South African Historical Journal, 35(1), 62-88. https://doi. org $/ 10.1080 / 02582479608671247$

Kozma, L. (2011). Cannabis prohibition in Egypt, 1880-1939: from local ban to League of Nations diplomacy. Middle Eastern Studies, 47(3), 443-460. https://doi.org/10.1080/00263206.2011.553890

López Restrepo, A. (2016). Remedios nocivos: los orígenes de la politica colombiana contra las drogas. Bogotá: IEPRI-Debate.

Lowes, P. D. (1966). The genesis of international narcotics control. Ginebra: Librairie Droz.

Mills, J. H. (2003). Cannabis britannica: empire, trade, andprobibition, 1800-1928. Oxford: Oxford University Press.

Mills, J. H. (2007). Colonial Africa and the international politics of cannabis: Egypt, South Africa and the origins of global control. En J. H. Mills \& P. Barton (Eds.), Drugs and empires: essays in modern imperialism and intoxication, c. 1500-c. 1930 (pp. 165-184). Basingstoke-New York: Palgrave Macmillan.

Mills, J. H. (2013). Cannabis nation: control and consumption in Britain, 1928-2008. Oxford: Oxford University Press.

Musto, D. F. (1999). The American disease. Origins of narcotic control ( $3^{\text {th }}$ ed.). New York-Oxford: Oxford University Press.

Nahas, G. G. (1982). Hashish in islam $9^{\text {th }}$ to $18^{\text {th }}$ century. Bulletin of the New York Academy of Medicine, 58(9), 814-831.

Paterson, C. (2009). Probibition \& resistance: a socio-political exploration of the changing dynamics of the Southern African cannabis trade, c. 1850-the present (Tesis de Master of Arts, Rhodes University, Grahamstown, Sudáfrica).

Rhenals Doria, A. M., \& Flórez Bolívar, F. J. (2013). Escogiendo entre los extranjeros "indeseables": afro-antillanos, sirio-libaneses, raza e inmigración en Colombia, 1880-1937. Anuario Colombiano de Historia Social y de la Cultura, 40(1), 243-271.

Rubim de Pinho, Á. (1975). Social and medical aspects of the use of cannabis in Brazil. En V. Rubin (Ed.), Cannabis and culture (pp. 293-302). La Haya-Paris: Mouton Publishers.

Schievenini Stefanoni, J. D. (2012). La probibición de la maribuana en México, 1920-1940 (Tesis de maestría, Universidad Autónoma de Querétaro, Querétaro, México). 
Schultes, R. E., Klein, W. M., Plowman, T., \& Lockwood, T. E. (1974). Cannabis: an example of taxomonic neglect. Botanical Museum Leaflets, 23(9), 337-367. Recuperado de http://www.jstor.org/stable/41762285 Shamir, R., \& Hacker, D. (2001). Colonialism's civilizing mission: the case of the Indian Hemp Drug Commission. Law \& Social Inquiry, 26(2), 435-461. https://doi.org/10.1111/j.1747-4469.2001.tb00184.x

Snelders, S., Kaplan, C., \& Pieters, T. (2006). On cannabis, chloral hydrate, and career cycles of psychotropic drugs in medicine. Bulletin of the History of Medicine, 80(1), 95-114. Recuperado de https:/ /www.jstor. org/stable/44449542

Stefanis, C., Ballas, C., \& Madianou, D. (1975). Sociocultural and epidemiological aspects of hashish use in Greece. En V. Rubin (Ed.), Cannabis and culture (pp. 303-326). La Haya-Paris: Mouton Publishers.

Taylor, A. H. (1969). American diplomacy and the narcotics traffic, 1900-1939: a study in international humanitarian reform. Durham: Duke University Press.

Willoughby, W. W. (1925). Opium as an international problem: the Geneva Conferences. Baltimore: The Johns Hopkins Press. 